NBER WORKING PAPER SERIES

\title{
A MODEL OF SOCIAL INTERACTIONS AND ENDOGENOUS POVERTY TRAPS
}

\author{
Roland G. Fryer, Jr. \\ Working Paper 12364 \\ http://www.nber.org/papers/w12364 \\ NATIONAL BUREAU OF ECONOMIC RESEARCH \\ 1050 Massachusetts Avenue \\ Cambridge, MA 02138 \\ July 2006
}

\begin{abstract}
Please direct all correspondence to the author at the following address: 1875 Cambridge Street; Cambridge, MA 02138. E-mail: rfryer@fas.harvard.edu. This paper circulated under the title "An Economic Approach to Cultural Capital." This work has greatly benefitted from thoughtful comments by Andrew Abbott, Susan Athey, Gary Becker, Douglas Bernheim, Edward Glaeser, Jerry Green, Michael Greenstone, Matthew O. Jackson, Lawrence Katz, William Johnson, Steven Levitt, Glenn C. Loury, Bentley MacLeod, Paul Milgrom, Kevin Murphy, Derek Neal, Peter Norman, Debraj Ray, Tomas Sjöström, and Chad Syverson. I would also like to thank seminar participants at CalTech, Chicago, Harvard, Notre Dame, NYU, Pennsylvania State, Stanford, UCLA, Virginia, and Wisconsin for their helpful comments and critiques. Lia Larson and Steven Ridgill provided exceptional research assistance. The author gratefully acknowledges financial support from NICHD and the National Science Foudation (SES-0109196). The usual caveat applies. The views expressed herein are those of the author(s) and do not necessarily reflect the views of the National Bureau of Economic Research.
\end{abstract}

(C2006 by Roland G. Fryer, Jr. All rights reserved. Short sections of text, not to exceed two paragraphs, may be quoted without explicit permission provided that full credit, including $@$ notice, is given to the source. 
A Model of Social Interactions and Endogenous Poverty Traps

Roland G. Fryer, Jr.

NBER Working Paper No. 12364

July 2006

JEL No. J0

\begin{abstract}
$\underline{\text { ABSTRACT }}$
This paper develops a model of social interactions and endogenous poverty traps. The key idea is captured in a framework in which the likelihood of future social interactions with members of one's group is partly determined by group-specific investments made by individuals. I prove three main results. First, some individuals expected to make group-specific capital investments are worse off because their observed decision is used as a litmus test of group loyalty - creating a tradeoff between human capital and cooperation among the group. Second, there exist equilibria which exhibit bi-polar human capital investment behavior by individuals of similar ability. Third, as social mobility increases this bi-polarization increases. The models predictions are consistent with the bifurcation of distinctively black names in the mid-1960s, the erosion of black neighborhoods in the 1970s, accusations of 'acting white,' and the efficacy of certain programs designed to encourage human capital acquisition.
\end{abstract}

\author{
Roland G. Fryer \\ Department of Economics \\ Harvard University \\ Littauer Center, M-7 \\ 1875 Cambridge Street \\ Cambridge, MA 02138 \\ and NBER \\ rfryer@fas.harvard.edu
}


"I got there [Holy Providence School in Cornwall Heights, right outside Philadelphia] and immediately found that I could read better than anyone else in the school. My father's example and my mother's training had made that come easy; I could pick up a book, read it aloud, pronounce the words with proper inflections and actually know what they meant. When the nuns found this out they paid me a lot of attention, once even asking me, a fourth grader, to read to the seventh grade. When the kids found this out, I became a target....

It was my first time away from home, my first experience in an all black situation, and I found myself being punished for everything I'd ever been taught was right. I got all A's and was hated for it; I spoke correctly and was called a punk. I had to learn a new language simply to be able to deal with the threats. I had good manners and was a good little boy and paid for it with my hide."

—Abdul-Jabbar, et. al., 1987, p.16.

\section{Introduction}

A century and a half after the treaty of Waitangi in New Zealand, six decades after Myrdal's account of race relations in the United States, and nearly a half century past Jim Crow in the U.S. and constitutionally recognized positive discrimination in India, social life around the world is characterized by significant racial and ethnic inequality. Many economic indices prove as much: wages, unemployment rates, income and wealth levels, standardized test scores, incarceration rates, and health and mortality statistics, all differ substantially across racial and ethnic categories.

An explanation that has garnered considerable attention from social scientists in discussions surrounding racial inequality in the US is that peers and communities impose costs on members who try to 'act white' (Fordham and Ogbu 1986, Austen-Smith and Fryer 2005). In this literature, minorities are seen as punishing other minorities for investing in behaviors that are deemed as the prerogatives for non-minorities (i.e. making good grades, listening to certain types of music, having non-minority friends, showing interest in classical music or ballet, and so on).

The limitations of the discussion, to date, is the microcosmic concentration on differences be- 
tween blacks and whites. ${ }^{1}$ If indeed there were an identifiable phenomenon, one would expect it to apply more generally. For example, in some Hispanic communities, women are discouraged from higher education and encouraged to be domesticated. In some traditional Amish communities, education is uniformly discouraged. In the Italian immigrant community in Boston's West End circa 1957, those who invested in education were labeled "mobiles" and "sissies." In the Latino community of greater Los Angeles, those who do not invest in sufficient amounts of local culture are labeled "Pocha." The Buraku of Japan either invest in their culture or disassociate themselves and attempt to achieve in the outside world. These latter examples do not occupy much space in the current literature involving racial and ethnic disparities, and more importantly, the trade-off between intra-group cooperation and economic success in the larger society. ${ }^{2}$

The literatures involving social stratification and inequality between and among racial groups in economics, sociology, and anthropology, unfortunately have different behavioral theories for each racial and ethnic group. These theories endeavor to explain differences in various social statistics (wages, unemployment rates, standardized test scores, and so on, e.g.) by bringing to bear contextual and group specific accounts that are rarely applicable to other minority groups. What is needed is a simple, empirically tractable, theoretical specification that is broad enough to encompass many diverse groups, but simple enough to have empirical content. This suggests a theory that encompasses the historic work on Italian immigrants in Boston (Gans 1962) and blacks on the South Side of Chicago in the 1930's (Drake and Cayton 1945) as well as recent work on 'acting white' (Fordham and Ogbu 1986, Fryer and Torelli 2005) and the ultra-orthodox Jews (Berman 2000), and applies more generally across many ethnic and racial groups.

With these ambitions in clear view, I present a simple model of social interactions and endogenous poverty traps that can be applied to many diverse communities. In particular, the theory applies to any group that requires its members to make costly community specific investments needed to facilitate local interaction with members of the community, but may run in conflict with economic success in the larger society. Examples of these communities include, but are not lim-

\footnotetext{
${ }^{1} \mathrm{Ogbu}(1986)$ is a notable exception.

${ }^{2}$ This is not meant to overlook the contributions by Bowles and Gintis (2004a, 2004b), Greif (1994), Iannacone (1992), Lazear (1999), and Berman (2000) which examine the intersection of culture and economic outcomes. The model to be presented is quite complementary to their work. There are, however, distinct empirical predictions which are highlighted in section 2.
} 
ited to, the Romany of Europe (Lee and Warren 1991), American Indians (Sowell 1994), Amish (Hostetler 1974), African-Americans (Fordham and Ogbu 1986), Hispanics (Fryer and Torelli 2005), West Indian immigrants (Waters 1999), the Maori of New Zealand (Ausubel 1977), the Sephardic Jews of Israel (Ogbu 1986), the Buraku of Japan (Devos and Wagatsuma 1966), Australian Aborigines (Altman and Nieuwenhuysen 1979), French Canadians, especially in Quebec and Montreal (Goyer 1983), and the coastal communities of Papua New Guinea.

Consider a stylized illustration of a community with many agents, set in Catalonia, Spain, which captures some of the basic ideas and intuitions. Each agent decides whether to invest their time in learning Catalan (a romance language spoken mainly by the local population) or computer programming. Investments in computer programming are valued in the global labor market, whereas, Catalan is only valued in the small local community. Agents observe each other's investment portfolio and can calculate the conditional probability of any agent being in the community in the future. Investments in Catalan yield a relatively high probability of being in the community in the future, since it is not valued elsewhere, and investments in computer programming yield a relatively low probability. The agents, then, play an infinitely repeated prisoner's dilemma with varying opponents, deciding whether or not to cooperate or defect in any given period, knowing their history of actions will be common knowledge. Cooperation, in this framework, can be interpreted as community interaction, the benefit of which is increasing in the time allocated to learning Catalan. In this stylized illustration, the prisoner's dilemma payoffs are quite natural. One can envision that agents will only cooperate if they observe sufficient investment in Catalan skills (i.e. the likelihood of being in the community in the future is relatively high).

Analyzing the model illuminates three basic categorizations of equilibria, the existence of which depends on particular parameter values. (1) There exist equilibria in which the most talented agents in a community refuse to invest in their group-specific capital in an effort to maximize their potential in the larger society, while less talented individuals invest in sufficient amounts to differentiate themselves from the high ability agents. This allows low ability agents to benefit from cooperation in lieu of labor market success. (2) There also exist equilibria in which everyone, regardless of innate ability, invests only in their group-specific capital, and refuse to invest in general human capital, even when they know that it is highly valued in the market. This is likely to happen when agents believe that the probability of interacting with members in their 
community in the future is highly non-responsive to investments in human capital. (3) There exists equilibria with the property that agents either invest all of their energies into human capital, or they do not invest anything. Analytically, this bipolarization follows from a highly non-concave objective function; optimal human capital investments do not vary continuously with ability. This leads to the unexpected conclusion that two agents, with slightly different innate ability, can have drastically different optimal human capital investments, and these differences are exaggerated as social mobility out of a community increases; a key prediction that differentiates the current model from previous work.

It is argued that the model's predictions are consistent with the rapid adoption of distinctively black names in the late 1960's, the bifurcation of black communities in the 1970's, the so-called 'acting white' phenomenon, and the efficacy of certain educational and job interventions designed to encourage investment among at-risk youth.

The theory provides a simple analytic framework that helps explain inter and intra group differences, without relying on differences in exogenously distributed tastes or innate ability. The only difference between groups (if any) is in the specificity of the group-specific investments needed to facilitate cooperation within a given community or group and its ability (or lack thereof) to stimulate human capital investments, and the likelihood of continued group interaction as a function of human capital acquisition.

Throughout the text, I give special emphasis to the education of African Americans in the U.S., though I envision much broader implications. This is not a theory narrowly tailored to the experience of African Americans. It is motivated by ethnographies highlighting similar behaviors of lower class communities across the world and endeavors to understand these behaviors in a simple equilibrium model.

insert figure 1

\section{A Model of Social Interactions and Endogenous Poverty Traps}

\section{A. Basic Building Blocks}

Let there be a continuum of agents with unit mass referred to as students and a finite set of 
agents referred to as (suitably anthropomorphized) communities. ${ }^{3}$ There are two types of communities: "poor" and "rich." For simplicity, we assume all agents begin in the poor community.

Nature moves first and distributes a type to each student. This type, denoted $\theta \in[\underline{\theta}, \bar{\theta}]$, represents a student's innate ability, where $\bar{\theta}$ denotes a student with relatively high ability, $\underline{\theta}$ denotes a student with relatively low ability; $F(\cdot)$ is the c.d.f. of $\theta$. Then, a student, knowing her private ability, makes a group-specific capital investment decision $c \in[0,1]$. Think of $c$ as the fraction of time that a student spends on group-specific investments - such as learning Catalan. ${ }^{4}$ Hence, $h=1-c$ denotes the student's human capital investment. ${ }^{5}$ The community, then, observes each student's group-specific capital investment, $c$, and plays an infinitely repeated prisoner's dilemma (hereafter referred to as the social interaction game) with the student, where both the student and the community decide whether to cooperate or defect in each period. This interaction can continue indefinitely, but can also end in any period with probability $(1-\delta(h))$, where $\delta:[0,1] \rightrightarrows[0,1]$ denotes the probability that a student with human capital $h$ will remain in the poor community in the subsequent period. ${ }^{6}$ Notice, $\delta(h)$ need not be monotonic.

\section{Strategies and Payoffs}

A strategy for a student specifies, for each type, the fraction of their time to be invested in human and group-specific capital, and a sequence of decisions in the social interaction game. To represent these strategies more formally, let $A=\{$ cooperate, defect $\}$ denote the set of realized choices in the social interaction game, with typical element $a^{j} \in A$, and let $\gamma^{0}$ denote the null history. Let $\Gamma^{t}=(A)^{t}$ be the space of possible period $t$ histories, and for $t \geq 1$, let $\gamma^{t}=\left(a^{0}, a^{1}, \ldots ., a^{t-1}\right)$ be a sequence of realized choices of actions at all periods before $t$. A strategy for a student, then, is a

\footnotetext{
${ }^{3}$ The conception that a student is interacting with a community may seem odd. This modeling strategy is a reduced form of a more general model in which there exists a large set of agents that are randomly grouped in pairwise matches in every period; if one assumes that the actions in any given match are common knowledge. Specifically, in a more elaborate model with random matching, any student, $i$, behaves as if he were facing the average characteristics of the set of agents with whom he is interacting. This is treated, formally, in an Appendix.

${ }^{4}$ Practical examples of these types of investments abound. In low-income black communities these investments might be in language or learning to be "streetwise" (Anderson 1992). In Italian immigrant communities, investments were in "the peer group society." (Gans 1962)

${ }^{5}$ This does not presume, a priori, that human capital and cooperation are necessarily in conflict with one another in one's community.

${ }^{6}$ The functional form of $\delta(h)$ is common knowledge among the agents.
} 
function $I:[\underline{\theta}, \bar{\theta}] \rightarrow[0,1]$ and a sequence of maps $\varphi_{s}^{t}: \Gamma^{t} \times[0,1] \times[\underline{\theta}, \bar{\theta}] \rightarrow[0,1]$. A community's strategy is a sequence of decisions in the social interaction game, denoted $\varphi_{n}^{t}: \Gamma^{t} \times[0,1] \rightarrow[0,1]$ for all $t$. I focus on "grim trigger" strategies. In words, any player who deviates from cooperation induces perpetual defection from the community. This is without loss of generality. If cooperation can be supported by any strategy, it can also be supported by grim trigger strategies.

\section{insert figure 2}

The stage game payoffs of the social interaction game are represented in figure 2. Consistent with the prisoner's dilemma, I assume $\alpha>0, \beta<0$, and $\mu>\alpha .^{7}$ There are several specifications of payoff functions found in the repeated game literature. I will focus on the case where players discount future utilities using $\rho \delta(h) \leq 1$, for all $h$, where $\rho$ represents a standard discount rate. ${ }^{8}$

Let $v_{j}(h, \theta)$ denote the value of being in community $j$ vis-à-vis of labor market earnings, conditional on being a type $\theta$ student who invested human capital $h ; v_{p}(h, \theta)\left(\right.$ resp. $\left.v_{r}(h, \theta)\right)$ denotes the value of being in a "poor" (resp. "rich") community. Poor and rich communities differ in myriad ways, including institutional infrastructure, social interactions and networks, and so on. I assume that $\frac{\partial v_{j}(h, \theta)}{\partial \theta}>0, \frac{\partial v_{j}(h, \theta)}{\partial h}>0, \frac{\partial v_{r}(h, \theta)}{\partial \theta}>\frac{\partial v_{p}(h, \theta)}{\partial \theta}$, and $v_{j}(h, \underline{\theta})$ is normalized to zero, all $h, j .{ }^{9}$ The basic idea is that simply moving to a rich neighborhood (weakly) raises labor market earnings, holding both ability and human capital constant, because of access to better social networks and opportunities.

\section{Equilibrium}

I shall focus on pure strategy equilibria, in which each agent makes a deterministic choice and all individuals of the same type make the same choice. The solution concept for this game, per

\footnotetext{
${ }^{7}$ In a more general setting, one might allow the payoff to cooperation to be a function of group-specific capital investments. A dynamic extension incorporating this is available from the author upon request.

${ }^{8}$ The social interaction game is, perhaps, the most ad hoc piece of the model and deserves further justification. It is meant to represent any social interaction that can be categorized as a costly public good. A concrete example is that of community policing or "watching each others back," which is described beautifully in Anderson's (1999) intimate portrayal of the social interactions among black youth on the west side of Philadelphia and Gan's (1962) description of the Italian immigrant community in Boston's West End.

${ }^{9}$ Similarly, if $\delta(\cdot)$ is a function of $\theta$, the same results are obtained-so long as $\delta(\cdot)$ is non-increasing in $\theta$. In this case, however, one must assume that the community knows the student's ability in order to solve the model.
} 
usual for repeated games, is Nash equilibrium. It is well known in the vast literature on repeated games that when the players are sufficiently patient, any finite gain from deviation of cooperation (defecting when the other player cooperates) is outweighed by even a small loss in utility in every future period, given the strategies are of grim trigger form. This implies, in the current framework, there exists $\delta^{*}$ such that for any $\delta(h) \geq \delta^{*}$, cooperation can be sustained. This is the subject of the first result.

Proposition 1 If $\delta(\cdot)$ is decreasing, grim trigger strategies constitute a cooperative Nash equilibrium of the infinitely repeated game if and only if $h \leq h^{*}$, where $h^{*}$ satisfies

$$
\delta\left(h^{*}\right)=\frac{1}{\rho}\left[1-\frac{\alpha}{\mu}\right]
$$

Proof. A student will deviate in any period $\tau \geq t$ if the expected discounted payoff from deviation is greater than the expected discounted returns for cooperating. The expected payoff for deviating in any period $t$ can be written as: $\mu+\frac{\delta(h) v_{p}(h, \theta)+(1-\delta(h)) v_{r}(h, \theta)}{(1-\rho \delta(h))}$. The expected discounted payoff from cooperation can be seen as: $\frac{\alpha+\delta(h) v_{p}(h, \theta)+(1-\delta(h)) v_{r}(h, \theta)}{(1-\rho \delta(h))}$. Therefore, the student wants to cooperate if and only if $\delta(h) \geq \frac{1}{\rho}\left[1-\frac{\alpha}{\mu}\right]$, which crosses once, given the assumptions on $\alpha(\cdot)$ and $\delta(\cdot)$.

Proposition 1 delineates communities into those in which human capital and local cooperation are diametrically opposed ( $\delta$ decreasing) and those in which it need not be. If, for example, $\delta$ is increasing (higher human capital implies a higher probability of future social interactions) only individuals with sufficient amounts of human capital can glean cooperation from the community.

\section{insert figure 3}

To be sure, there is "nerd bashing" behavior in most neighborhoods, irrespective of race or cultural affiliation. Yet, the tolerable level of human capital $\left(h^{*}\right)$ can be quite different across communities. ${ }^{10}$ Consider figure 3 , which illustrates a plausible $\delta$ for a hypothetical middle income neighborhood. It assumes that those with extreme levels of human capital (high or low) are likely to leave. Students in these communities cannot benefit from cooperation if they invests too little (are

\footnotetext{
${ }^{10}$ In a more elaborate model in which the severity of punishment depends on the size of the group who are not investing, punishments across neighborhoods could also be quite different - further pushing down $h^{*}$
} 
likely to move to poor neighborhoods) or too much (are likely to move to rich neighborhoods). In this case, the student cooperates in social interactions only if he invests an interior $h \in[\underline{h}, \bar{h}]$. So, students in middle class neighborhoods can face disincentives of investing in human capital similar to those endured by students in low income neighborhoods. Again, however, $h^{*}$ will differ between these communities as $\delta(\cdot)$ differs. This captures a very nice feature of the model which warrants further emphasis. The model of social interactions is flexible enough to investigate antagonizing behavior and under investment in education in many communities. The critical parameter is $\delta(\cdot)$.

Let $c^{*} \equiv 1-h^{*}$, where $h^{*}$ is defined by equation (1). If the community observes $c \geq c^{*}$, cooperation can be sustained and if they observe $c<c^{*}$ it cannot. For simplicity and transparency, I assume that the community (resp. student) will cooperate if and only if $c \geq c^{*}{ }^{11}$ Thus, the student knows if he invests $c \geq c^{*}$ in his group-specific capital, his peers will cooperate, and if not they won't. Let $\widehat{\alpha}=\alpha$ for all $h \leq h^{*}$, and $\widehat{\alpha}=0$ for all $h>h^{*}$. With this, the student's expected utility can be readily derived. Once a student makes her group-specific capital decision, we know $\delta(h), v_{p}(h, \theta)$, and $v_{r}(h, \theta)$. Her utility in period one is comprised of her social payoff $(\widehat{\alpha})$ and her economic payoff $\left(v_{j}(h, \theta)\right)$, where the latter depends on her exit probability. In symbols, her expected payoff in period one is $\widehat{\alpha}+\delta(h) v_{p}(h, \theta)+(1-\delta(h)) v_{r}(h, \theta)$. Recall, with probability $\delta(h)$ she stays in her community. Thus, if she stays in the game two periods, her two-period expected payoff can be written as

$$
\widehat{\alpha}+\delta(h) v_{p}(h, \theta)+(1-\delta(h)) v_{r}(h, \theta)+\rho \delta(h)\left[\widehat{\alpha}+\delta(h) v_{p}(h, \theta)+(1-\delta(h)) v_{r}(h, \theta)\right]
$$

Following this logic over an infinite horizon, the student's expected utility can be written as:

$$
\widehat{U}(h, \theta) \equiv \frac{\widehat{\alpha}+\delta(h) v_{p}(h, \theta)+(1-\delta(h)) v_{r}(h, \theta)}{1-\rho \delta(h)} .
$$

This leads to the following concise characterization of equilibrium student behavior.

Proposition 2 In any cooperative Nash equilibrium, a type $\theta$ student chooses $\max \{\widehat{U}(\widehat{h}, \theta), \widehat{U}(1, \theta)\}$, where

$$
\widehat{h}(\theta) \equiv \underset{h \in\left[0, h^{*}\right]}{\arg \max }\left\{\frac{\alpha+\delta(h) v_{p}(h, \theta)+(1-\delta(h)) v_{r}(h, \theta)}{1-\rho \delta(h)}\right\}
$$

\footnotetext{
${ }^{11}$ There are many other equilibria in the social interaction game, even when agents are patient. However, cooperation yields the payoff dominant equilibrium in the infinitely repeated prisoner's dilemma.
} 
Proof. The community's choice problem is trivial: cooperate if and only if they observe $c \geq c^{*}$. Expecting this, any student who optimally invests $c<c^{*}$, chooses $c=0(h=1)$, since they derive no benefit from cooperation and cultural capital is costly. Let $\widehat{h} \equiv \underset{h \in\left[0, h^{*}\right]}{\arg \max }\left\{\frac{\alpha+\delta(h) v_{p}(h, \theta)+(1-\delta(h)) v_{r}(h, \theta)}{1-\rho \delta(h)}\right\}$ denote the student's optimal level of human capital, conditional on ensuring cooperation from the community. The students' choice problem can then be summarized as choosing to either: "opt for cooperation" $(h=\widehat{h})$ or "opt out" $(h=1)$. In symbols, the student chooses $\max \{\widehat{U}(\widehat{h}, \theta), \widehat{U}(1, \theta)\}$.

Proposition 2 provides a simple dichotomous solution to a complicated decision problem. Students either opt out - investing all of their time in human capital in an effort to maximize their utility, or opt for cooperation - under investing in human capital (relative to their optimum in a community without the social interaction constraints) to assure cooperation from their community and receive the social benefits.

There are two cases to consider, which depend on how the student's utility function varies with $h$ when he opts for cooperation. For convenience, let $M B(h ; \theta)$ denote the marginal benefit of investing an additional unit in human capital, for a talent $\theta$ student, when a student opts for cooperation, and $M C(h ; \theta)$ denote the marginal cost. ${ }^{12}$ The marginal benefit of investing an additional unit of human capital, when the student opts for cooperation, is comprised of two parts. First, additional human capital increases the value of being in both communities. Second, when a student in the poor community invests more, he increases the likelihood of exit - yielding higher expected payoff. On the other hand, the marginal cost of investing in $h$ consists of the lower likelihood of receiving utility $\alpha$ from the social interaction game and the value $v_{p}(h, \theta)$. This is the basic economics of the student's investment decision.

\section{insert figure 4}

Suppose that the objective function is increasing in human capital for all students who opt for cooperation $\left(M B(h ; \theta)>M C(h ; \theta)\right.$ for all $\left.h \in\left[0, h^{*}\right]\right)$. This will be the case when the payoff to cooperation in social interactions, $\alpha$, is sufficiently small. In this scenario, figure 4 represents the students choice problem. We know that any student who opts out chooses $h=1$, and any student

\footnotetext{
${ }^{12} M B(h ; \theta)$ and $M C(h ; \theta)$ are derived by differentiating $\widehat{U}(h, \theta)$ with respect to $h$ and taking the positive elements of the resulting equation to be $M B(h ; \theta)$ and the negative elements to be $M C(h ; \theta)$.
} 
who opts for cooperation chooses $h=\widehat{h}$. These points are labeled in figure 4 . Whether or not a student chooses $h=\widehat{h}$ or $h=1$, depends on his ability $(\theta)$. In particular, figure 4a represents a student with relatively low $\theta$ and figure $4 \mathrm{~b}$ represents a student with relatively high $\theta$. One can envision, then, situations in which large differences in human capital can occur among individuals in the same community who have very similar innate ability. Given the continuity assumptions and noting that the marginal value of opting out is increasing in $\theta$, relative to opting for cooperation, we know there exists a student of ability $\theta^{*}$ who is just indifferent between investing a lot $(h=1)$ or investing substantially less $(h=\widehat{h})$. Formally,

$$
\theta^{*} \text { solves } \frac{\alpha+\delta(\widehat{h}) v_{p}\left(\widehat{h}, \theta^{*}\right)+(1-\delta(\widehat{h})) v_{r}\left(\widehat{h}, \theta^{*}\right)}{1-\rho \delta(\widehat{h})}=\frac{\delta(1) v_{p}\left(1, \theta^{*}\right)+(1-\delta(1)) v_{r}\left(1, \theta^{*}\right)}{1-\rho \delta(1)} .
$$

The left hand side of (4) is strictly greater than the right hand side for $\theta$ sufficiently small. This, coupled with the fact that the marginal value of an increase in $\theta$ is greater for those who opt out, proves the existence of such a $\theta^{*}$. Now consider the student who has ability $\theta^{*}-\varepsilon$. In this case, an agent with slightly less ability, invests substantially less in human capital.

Let

$S=\left\{\theta \in[\underline{\theta}, \bar{\theta}]: \frac{\alpha+\delta(\widehat{h}) v_{p}(\widehat{h}, \theta)+(1-\delta(\widehat{h})) v_{r}\left(\widehat{h}, \theta^{*}\right)}{1-\rho \delta(\widehat{h})}=\frac{\delta(1) v_{p}\left(1, \theta^{*}\right)+(1-\delta(1)) v_{r}\left(1, \theta^{*}\right)}{1-\rho \delta(1)}\right\}$

denote the set of ability types that opt for cooperation. Notice: if the probability of being in the community in the following period $(\delta(\cdot))$ is relatively inelastic (i.e., relatively non-responsive to $h$ ); then the equilibrium exhibits bipolar investment behavior with small differences in innate ability, leading to large human capital differences. These are qualitative predictions that cannot be found in current models of social interaction.

\section{insert figure 5}

Things are quite different when the student's objective function is decreasing in human capital for those who opt for cooperation $\left(M B(h ; \theta)<M C(h ; \theta)\right.$ for all $\left.h \in\left[0, h^{*}\right]\right)$, which is the case when $\alpha$ is sufficiently large. Two prototypical examples are depicted in figure 5. This produces an even stronger endogenous bipolarization; students in effect choose $h \in\{0,1\}$. It is imperative to note, however, that if the model were extended to allow students to decide whether or not to leave once 
they received an offer in the rich neighborhood, this case would be eliminated. With this extension, students would opt for cooperation and invests $\widehat{h}=h^{*}$, even if $\alpha$ were large because they would simply choose to stay even if offered a job outside the community and it would be optimal to choose $\widehat{h}=h^{*}$, since $\frac{\partial v_{p}(h, \theta)}{\partial h}>0$. For this reason, I hereafter concentrate on equilibrium in which $\widehat{h}=h^{*}$.

Suppose there are two communities: community 1 and community 2, with corresponding exit probabilities $\delta_{1}$ and $\delta_{2}$. In both communities let there be a negative relationship between investment in human capital and the likelihood of future interactions with the community, though in community 2, allow the slope to be steeper. So, community 2 can be thought of as a "tougher" neighborhood. The following result shows that agents who opt for cooperation in community 2 (the "tougher" neighborhood) will actually invest more in human capital than those who opt for cooperation in community 1 . In other words, increasing the porosity between neighborhoods has the unintended effect of increasing the fragility of cooperation in social interactions. The fraction of students, however, who opt for cooperation in community 2 is smaller. And, for any student who would opt for cooperation in either community - he would invest more in human capital in the tougher neighborhood.

\section{insert figure 6}

Proposition 3 If $\delta_{1}(h)>\delta_{2}(h)$ for all $h \in\left[0, h^{*}\right]$, then $S_{2} \subseteq S_{1}, \bar{h}_{1} \geq \bar{h}_{2}$, and $\int_{S_{2}} \widehat{h}_{1}(\theta) d F(\theta)>$ $\int_{S_{2}} \widehat{h}_{2}(\theta) d F(\theta)$ where $\bar{h}_{i}=\int_{S_{i}} \widehat{h}(\theta) d F(\theta)$.

Proof. We know from equation (1) that if $\delta$ increases, $h^{*}$ decreases, and subsequently, $c^{*}$ increases. It follows directly that $S_{1} \supseteq S_{2}$. To specifically extrapolate the implications of this effect, see figure 5. In this, the agents whom opt for cooperation (depicted in (A)), must choose a lower level of human capital $\widehat{h}=h^{* *}$ in order to gain cooperation. In this sense, increasing the fragility of the relationship by increasing the probability that one can leave the community has the effect of suppressing the human capital of those who opt for cooperation. However, as pictured in case (B), sufficiently talented agents are not effected by this, given they invest all of their energies into human capital, regardless. The Proposition follows directly.

Proposition 3 is a key result that differentiates the current model from previous work at the intersections of social capital, culture, and economics. It provides a very striking and unique prediction: when social mobility between neighborhoods increases, agents in poor neighborhoods will 
invest even more in their local culture - as it serves as a strong signal of their group committment.

In what follows the model is applied in a myriad of applications and its predictions discussed.

\section{Understanding Black Progress in the US Through the Lens of the Model}

In this section, I explore a variety of examples and applications of the basic theory. Although it is simple, its empirical predictions and testable implications are quite consistent with several important facts concerning Black progress in the US: the adoption of distinctively Black names, the decline of black neighborhoods in the 1970's, educational achievement, and the efficacy of particular educational and job interventions programs.

Distinctive Black Names

insert figure 7

The fundamental predictions to test from the social interaction model, that will allow one to differentiate it from other models of social capital and culture (Berman 2000, Borjas 1992 and 1995, Glaeser, Laibson, and Sacerdote 2002, Iannocone 1992, and Lazear 1999), are the predictions in Proposition 3 concerning agents equilibrium responses to exogenous changes in social mobility. A nice test of these predictions is to focus on one dimension of investment in group specific capital- the distinctive choice of first names. The model predicts that as the porosity between neighborhoods increases, names of those in black neighborhoods (who opt for cooperation) will get unambiguously blacker while names of blacks in white neighborhoods (who opt out) will get whiter - creating a bifurcation in the distribution of black names.

Figure 7 provides empirical evidence to this effect, demonstrating a striking time series pattern that shows a bifurcation in the distribution of African American first names, circa $1968 .{ }^{13}$ Two years after the Fair Housing Act, which reduced the barriers to integration, blacks living in

\footnotetext{
${ }^{13}$ The data used are drawn from the Birth Statistical Master File maintained by the Office of Vital Records in the California Department of Health Services. These files provide information drawn from birth certificates for virtually all children born in California over the period 1961-2000, which has been supplemented with personal identifiers including mother's first name, mother's maiden name, and child's full name.
} 
predominantly black neighborhoods start to adopt distinctively "black" names, whereas blacks living in predominantly white neighborhoods adopt traditional white names. The numbers are quite startling. The median black female in a predominantly black area went from receiving a name that was twice as likely to be Black (before the Fair Housing Act) to a name that was more than twenty times as likely to be given to Blacks. Among Blacks in integrated neighborhoods, no such transformation occurred, with Black names actually becoming more similar to whites. These data provide a direct test of the model, if one assumes that the choice of a first name is a group-specific investment decision.

The weakness in the argument, however, is that Fryer and Levitt (2004) find no causal impact of names on adult outcomes. In order for names to serve as a commitment device, they need to represent a costly investment. This can be easily reconciled in that names are just one dimension one can signal their group loyalty.

\section{Historical Applications}

From American Chattel slavery through Jim Crow, social mobility among Blacks was effectively zero. During this time, investing in human capital was not seen as a threat of leaving the community because $\delta(\cdot)$ was highly inelastic, due to institutional barriers. In a typical black community, doctors, lawyers, postmen, and others with lower occupational status, lived in the same vicinity. With the decrease in institutional discrimination and the increase in housing integration came many new opportunities, including the choice of moving to a more integrated neighborhood.

Game theoretically, this integration changed the game from a infinitely repeated game in which the probability of future interactions other individuals was near one, to a game in which agents leave with a probability that depends on their human capital investments. With this change, community monitoring of agents' human capital became an important predictor of their future behavior.

In his classic portrayal of neighborhoods in Chicago, Wilson (1978) argues that the African American community was splitting into two, with middle class blacks increasing their position relative to whites, and poor blacks becoming even more marginalized. Wilson's conjecture is that the plight of the black inner cities was due to the erosion of their social networks and social capital. This element of self-selection is readily seen in the framework presented here, as $\delta(\cdot)$ changes over time. There are, however, some subtle differences. Whereas Wilson argues that networks are to 
blame, I argue that the very presence of high ability blacks and institutional barriers allowed those on the margin to invest more in human capital without loosing cooperation.

\section{Policy Interventions}

A myriad programs seek to differentiate and improve the educational environment for innercity or poor youth by on-site or remote training. To date, most of the focus on educational interventions have been on early childhood development. Model programs of this kind include the Milwaukee Project (Garber 1988), Early Training Project (Gray et. al. 1983), High/Scope Perry Pre-school Project (Schweinhart, et. al. 1993), and the Carolina Abecedarian Project (Campbell and Ramey 1994). There are fewer programs that target secondary school students. Interventions include gifted and talented programs within schools and counseling/mentoring programs. Offering separate courses or co-curricular organizations for high achieving students is equivalent to adding non-random matching into the basic model in an effort to induce sorting on ability. Offering remote training or moving kids out of their neighborhoods can also induce sorting, or more importantly, change the functional form of $\delta(\cdot)$ depending on which type of program is pursued. I discuss each of these programs in turn within the context of the basic model and tangential extensions.

Honors courses, co-curricular activities (i.e. math club, upward bound, etc.), and counseling/mentoring programs are a common component of many secondary schools. A simple extension of the basic model allows us to investigate the implications of such interventions in particular communities where the social interaction game is being played.

Consider the following extension of the basic model. Students can choose to join one of two groups: the cool group or the smart group, with an epsilon cost of joining the cool group. After observing their talent, students choose their group-specific investment, $c$, and are randomly matched with members within their group with probability $\gamma>\frac{1}{2}$. Students' expected payoff of investing $c_{i}$, in the social interaction game, if they choose to cooperate is then $\alpha$. Solving the model with this simple extension proves that all individuals in the smart group invest $h=1$ and all individuals in the cool group invest $h=\widehat{h}$. This is an equilibrium, since no cool students want to join the smart group, given they will get no value out of the social interaction game, and no smart students want to join the cool group-given the epsilon cost. ${ }^{14}$ Indeed, in some cases, all agents are weakly better

\footnotetext{
${ }^{14}$ This follows from the fact that there are no human capital externalities in the model from interacting with
} 
off under this assortative matching type equilibrium.

Remote training programs are another form of educational intervention for secondary school students, though less common. They involve taking students out of their classroom environments and engaging them in educational programs away from their communities. This usually takes one of two forms: students are removed from their community for a fixed amount of time (day, summer, etc.), or students are removed from their community permanently. The former characterization is equivalent to the sorting type equilibrium previously described. Versions of the latter characterization include the Gautreaux program, a major initiative adopted by the courts to provide a metropolitan-wide remedy for racial discrimination in Chicago's public housing, Movingto-Opportunity, Job Corps, and the well known A Better Chance Program ${ }^{15}$. In the A Better Chance initiative, students leave their families and live with a host family to attend better schools. Consider the following quote from a student in the A Better Chance Program.

"I felt I could be more involved with my studies here [in the host family]. At home, I would be distracted by peer pressures to hang out, smoke and drink. Here, I can focus on the academics. You face peer pressure wherever you go, but at Radnor there are more kids into their studies. ${ }^{16 "}$

The social interaction model predicts that these types of interventions, will have larger marginal effects on students' educational achievement because they change $\delta(\cdot)$. Other interventions induce sorting, but have an ambiguous effect on the marginal student. The available evidence suggests that this is indeed the case. Sixty-five percent of the students in the A Better Chance program come from single parent families and thirty-three percent of them are beneath the poverty threshold; however, ninety-nine percent of the A Better Chance seniors immediately enroll in college. This is significantly larger than any other secondary educational intervention. However, there are thorny selection issues to consider before one can test the programs causal impacts.

More convincing are the results from analysis of the Job Start and Job Corps programs. Job Corps is the nation's largest and most comprehensive residential, education and job training prostudents in the smart group. In general, we know that learning begets learning (see Heckman, 2000). This complicates matters, but does not take away from the qualitative insights of the result.

${ }^{15}$ To read more about this program, visit http: \\www.abetterchance.org

16 "Going 'Away' to School in Radnor: A Better Chance for Teens Who Put Their Schooling First." The Suburban and Wayne Times, September 23, 1999. 
gram for at-risk youth, ages 16 through 24 . It takes the students to (predominantly rural) training centers where they receive free room and board along with intense training in one of 100 vocational specializations. Conversely, Job Start uses the same teaching curricula as Job Corp, but the students stay at home and commute to a local training site. As the model would predict, Job Corps has larger effects. It has been shown that Job Corp increases earnings and reduces crime, whereas, Job Start has shown statistically insignificant effects. ${ }^{17}$

Essentially, the aim of any educational intervention, mindful of the type of peer effects described here, must be geared toward changing $\delta(\cdot)$ or decreasing $\alpha$-which have different implications for policy depending on which is pursued. Changing $\delta(\cdot)$ literally involves moving kids from low income neighborhoods to suburbia or changing the nature of interaction between high achieving students who get out and those who are left behind. Conversely, changing $\alpha$ involves minimizing social interactions and the development of peer groups within schools.

A nice test of the efficacy of the latter policy would be to investigate the black-white achievement gap among military children at military schools, where presumably, racial differences in cultural capital are minimal. The theory predicts that these gaps will be substantially smaller. As evidence to this effect, Brown (2003) shows that white children from military families score slightly higher than do their civilian counterparts, while black children from military families do significantly better than black children from non-military families. The test score gap is about 40 percent smaller in the military than in civilian schools.

\section{Conclusion}

Blacks antagonize fellow blacks for not being "black enough," whites ridicule other whites for "trying to act black," and Italian immigrants mock other Italian immigrants for being "sissies." Some observe these behaviors and conclude that those who ridicule others are punishing them because they have formed an oppositional culture against certain behaviors of other cultures, have an intrinsic preference for seeing them suffer, or some unspecified social forces have led to such behavior. The economic explanation, however, is quite different.

By using a simple model of social interactions with an endogenous probability that the re-

\footnotetext{
${ }^{17}$ See http://www.jobcorps.org and http://www.mdrc.org/project_9_60.html for results on Job Corp and Job Start programs, respectively.
} 
lationship will end in any period, cooperation can only be sustained with those members of the community who they are sufficiently likely to interact with in the future. There is no preference based punishment mechanism needed, just rational defection when an agent's probability of being around in the future is too low. This causes agents in these communities to optimally trade off cooperation with community and economic success. Using this simple approach, I have shown that some individuals subject to group-specific investments are worse off because these observable investments are used, in equilibrium, as a litmus test of group loyalty. I have also shown that as the value of human capital increases, the inter and intra group inequality can increase. This is a direct result of the discontinuity and non-concavity in the student's objective function. Further, as social mobility increases the cultural capital phenomenon should become even more profound and not, as the leading sociological theory would suggest, die out.

One shortcoming of the current approach is that defection in the repeated game may not coincide with the active antagonistic behavior that motivated the present study. There are many types of social interactions at work in communities, simultaneously, and I have chosen to highlight one. How this fits into a larger framework of group dynamics and collective identity is left for future work.

\section{References}

[1] Abdul-Jabbar, Kareem, Knobler, Peter, and Knobler, Jerry. 1987. Giant Steps. Bantam Books

[2] Altman, Jon and Nieuwenhuysen, John. 1979. The Economic Status of Australian Aborigines. (Cambridge: Cambridge University Press).

[3] Anderson, Elijah. 1992. Streetwise: Race, Class, and Change in an Urban Community. Chicago: University of Chicago Press.

[4] Anderson, Elijah. 1999. Code of the Street: Decency, Violence, and the Moral Life of the Inner City. Norton, W. W. \& Company.

[5] Ausubel, David. 1977. Maori Youth: A Psychoethnological Study of Cultural Deprivation. (Massachusetts: The Christopher Publishing House).

[6] Austen-Smith, David and Roland Fryer. 2005. "An Economic Analysis of 'Acting White'," Quarterly Journal of Economics, 120,2, 551-583. 
[7] Berman, Eli. 2000. "Sect, Subsidy, and Sacrifice: An Economist's View of Ultra-Orthodox Jews." Quarterly Journal of Economics, 115(3), 905-953.

[8] Borjas, George. 1992. "Ethnic Capital and Intergenerational Mobility." Quarterly Journal of Economics, 107 (1) p. 123-50.

[9] Borjas, George. 1995. "Ethnicity, Neighborhoods, and Human-Capital Externalities." American Economic Review, 85, 3, p. 365-90.

[10] Bowles, Samuel and Gintis, Herbert. 2004a. "The Evolution of Strong Reciprocity." Theoretical Population Biology, 65, 17-28.

[11] Bowles, Samuel and Gintis, Herbert. 2004b. "Persistent Parochialism." Journal of Economic Behavior and Organization, 55, $1-23$.

[12] Brown, Charles. 2003. "Relatively Equal Opportunity in the Armed Forces: Impacts on Children of Military Families." Working Paper. University of Michigan.

[13] Campbell, Frances, and Ramey, Craig. 1994. "Effects of Early Intervention on Intellectual and Academic Achievement: A Follow-up Study of Children from Low-income Families." Child Development. 65, pp. 684-98.

[14] Devos, George, and Wagatsuma, Hiroshi. 1966. Japan's Invisible Race: Caste in Culture and Personality. University of California Press.

[15] Drake, St. Clair., and Cayton, Horace. 1945. Black Metropolis: A Study of Negro Life in a Northern City. Chicago: University of Chicago Press.

[16] Fordham, Signithia and Ogbu, John. 1986. "Black Students' School Successes: Coping with the Burden of 'Acting White'," The Urban Review 18 (3): 176-206.

[17] Fryer, Roland and Levitt, Steven. 2004. "The Causes and Consequences of Distinctively Black Names." The Quarterly Journal of Economics, 119 (3), 767-805.

[18] Fryer, Roland and Torelli, Paul. “An Empirical Analysis of 'Acting White.' NBER Working Paper No. 11334. 
[19] Gans, Herbert. 1962. The Urban Villagers: Group and Class in the Life of Italian-Americans. New York: The Free Press

[20] Garber, H.L. 1988. The Milwaukee Project: Prevention of Mental Retardation in Children at Risk. Washington D.C.: American Association on Mental Retardation.

[21] Glaeser, Edward., Laibson, David., and Sacerdote, Bruce. 2002. "An Economic Approach to Social Capital." Economic Journal, 112, p. 437-458.

[22] Goyer, John. 1983. Ethnicity and Class Identity: The Case of French and English Speaking Canadians.

[23] Gray, S.W., Ramsey, B., and Klaus, R., 1983. "From 3 to 20: The Early Training Project," in As the Twig is Bent....Lasting Effects of Preschool Programs. Consortium for Longitudinal Studies, ed. Hillsdale N.J.: Erlbaum, pp. 171-200.

[24] Greif, Avner. 1994. "Cultural Beliefs and the Organization of Society: An Historical and Theoretical Reflection on Collectivist and Individual Societies." Journal of Political Economy, $102(5)$, pp. $912-50$

[25] Heckman, James. 2000. "Policies to Foster Human Capital." Research in Economics. 54. pp $3-56$.

[26] Hostetler, John. 1974, "Education in Communitarian Societies: The Older Amish and the Hutterian Bretheren." in Educational and Cultural Process: Toward and Anthropology of Education, George Spindler, Ed. (New York: Holt, Rinehart, and Whinston).

[27] Ianaccone, Laurence. 1992. "Sacrifice and Stigma: Reducing Free-Riding in Cults, Communes, and Other Collectives." Journal of Political Economy, 100 (2), pp. 271-91.

[28] Lazear, Edward. 1999. "Culture and Language." Journal of Political Economy, 107 (6), S95S126.

[29] Lee, K.W. and Warren, W.G. 1991. "Alternative Education: Lessons from Gypsy Thought and Practice" British Journal of Educational Studies 39 (3), 311-324. 
[30] Ogbu,. John. 1986. "The Consequences of the American Caste System" in The School Achievement of Minority Children, Ulric Neisser, ed.

[31] Schweinhart, Lawrence, and Barnes, Helen, and Weikart, David. 1993. Significant Benefits: The High/Scope Perry Preschool Study Through Age 27. Monograph of the High/Scope Educational Research Foundation, 10, Ypsilanti, Michigan: High/Scope Educational Research Foundation.

[32] Sowell, Thomas. 1994. Race and Culture: A World View. (New York, Basic Books).

[33] Waters, Mary. 1999. Black Identities: West Indian Immigrant Dreams and Realities. (New York: Russel Sage Foundation).

[34] Wilson, William-Julius. 1978. The Declining Significance of Race: Blacks and Changing American Institutions. The University of Chicago Press. 
Nature Distributes Students' Types
Students Observe Type and

Choose Cultural Investment
Community Observes

Cultural Investment
Play Social Interaction Game

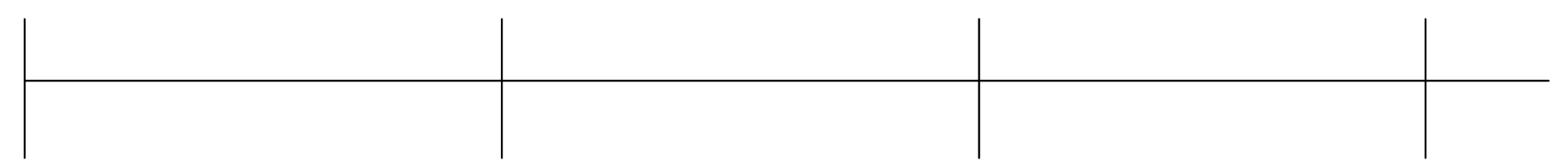

Figure 1 


\section{$\underline{\text { Payoffs }}$}

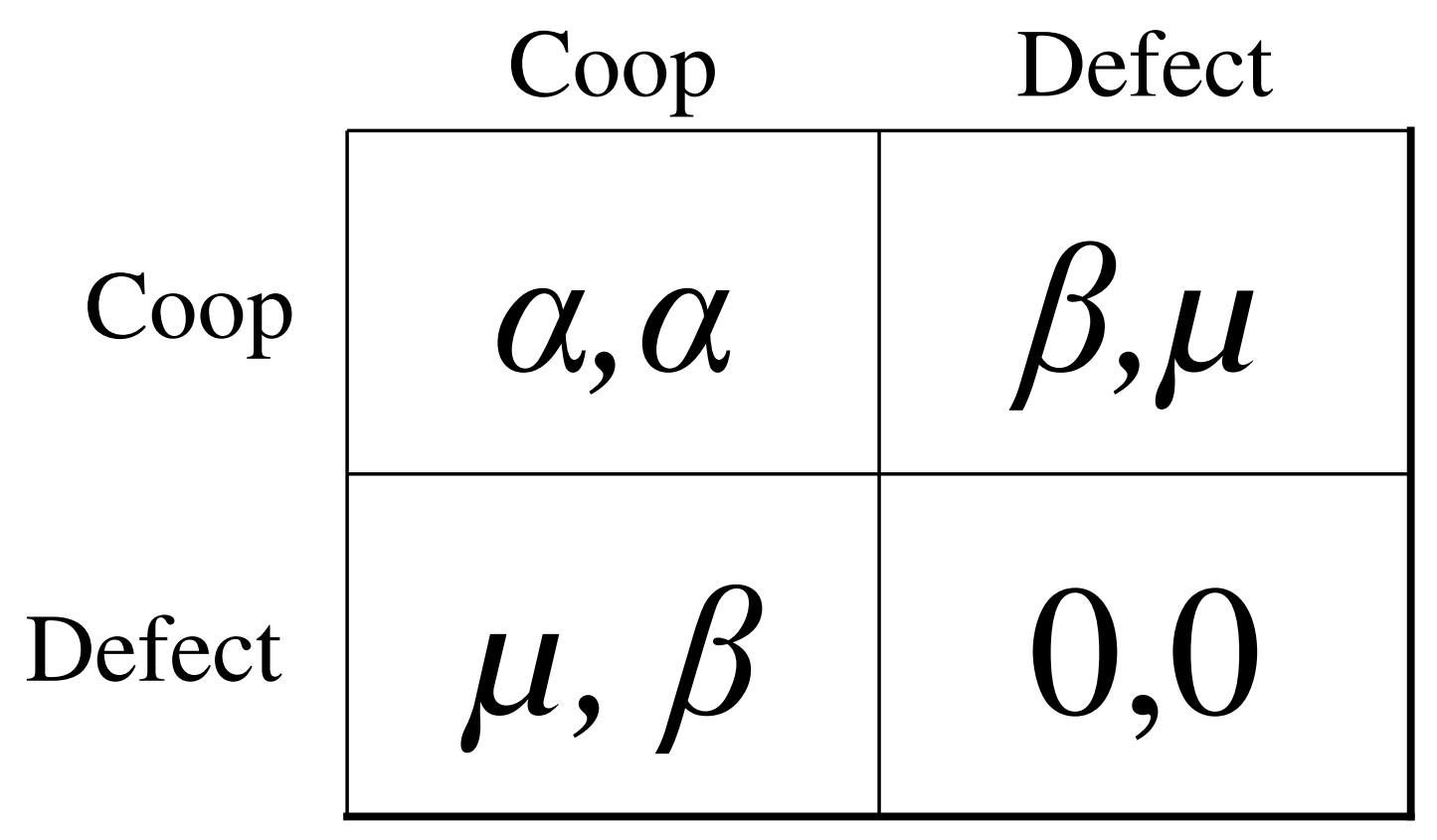

Figure 2 


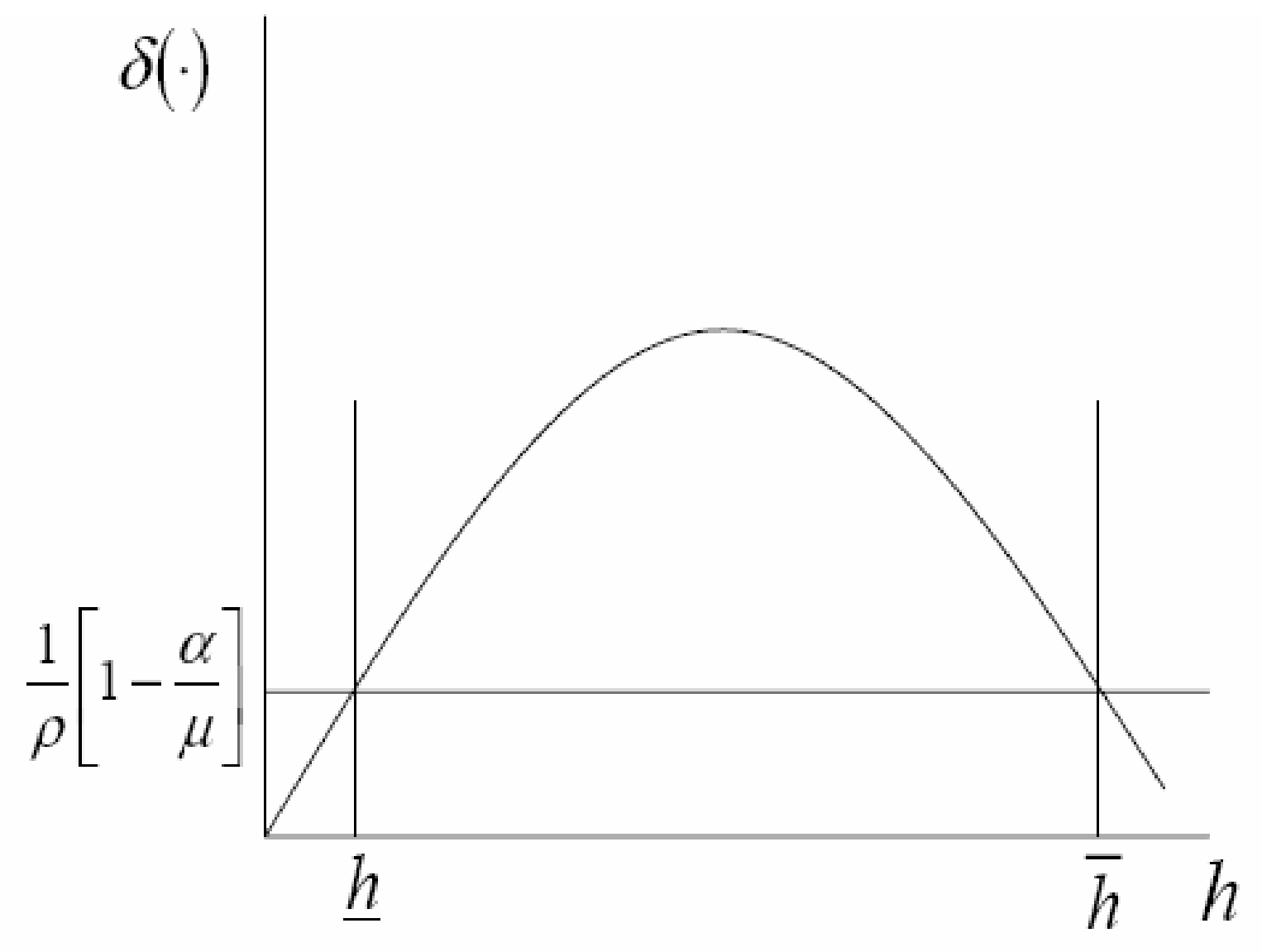

Figure 3 


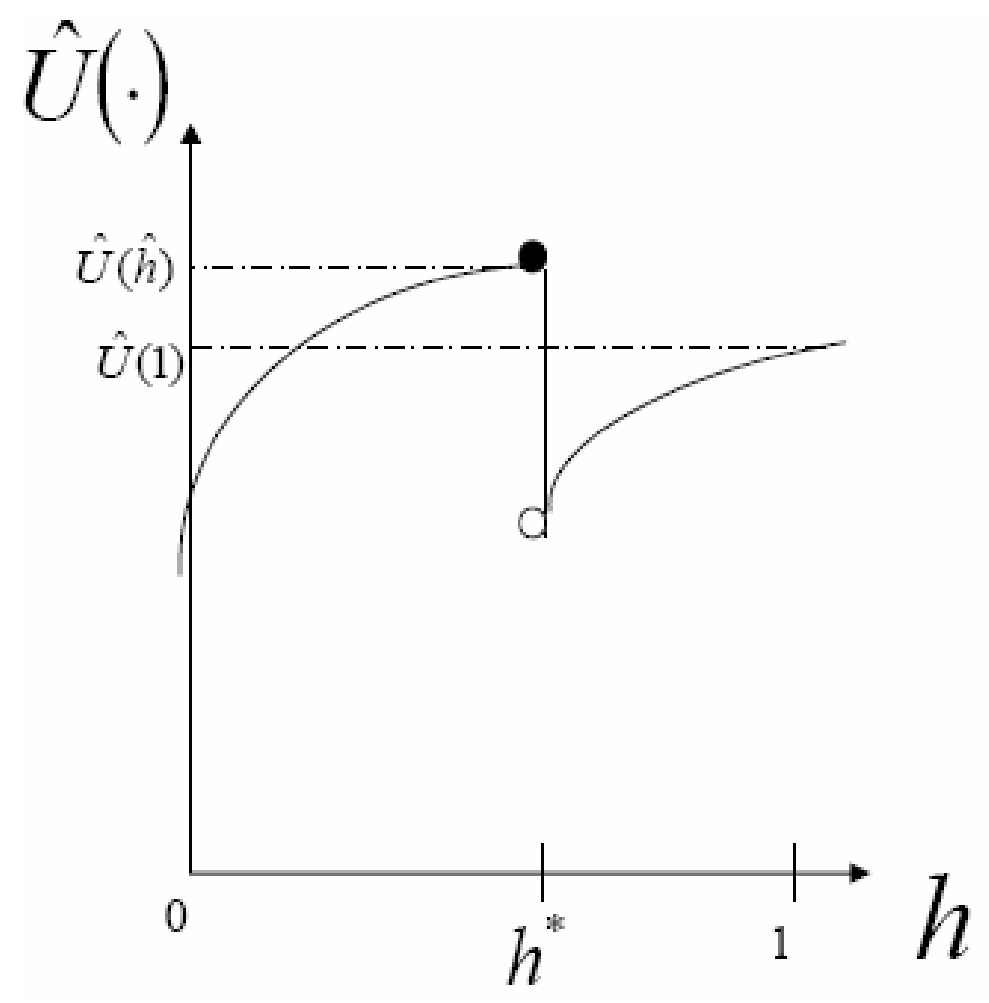

Low Ability

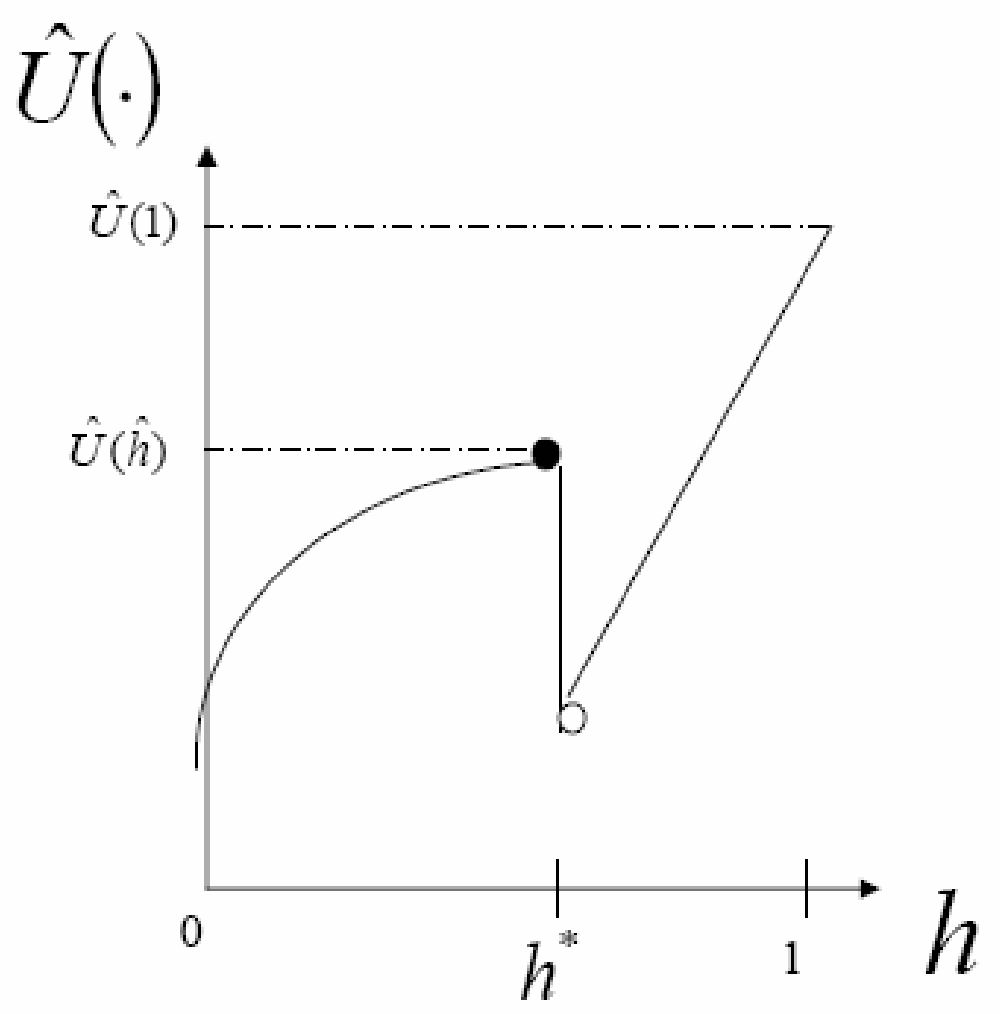

High Ability

Figure 4 


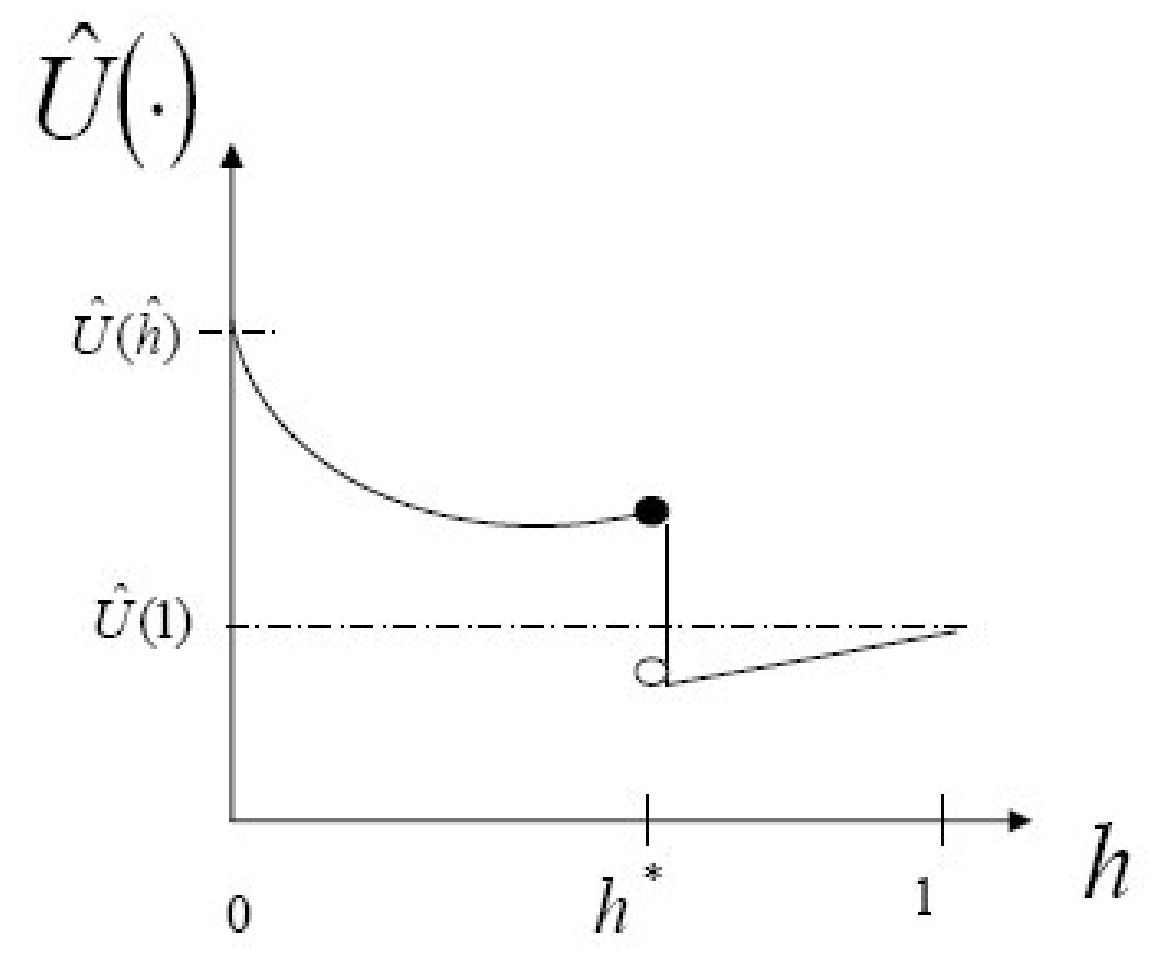

Low Ability

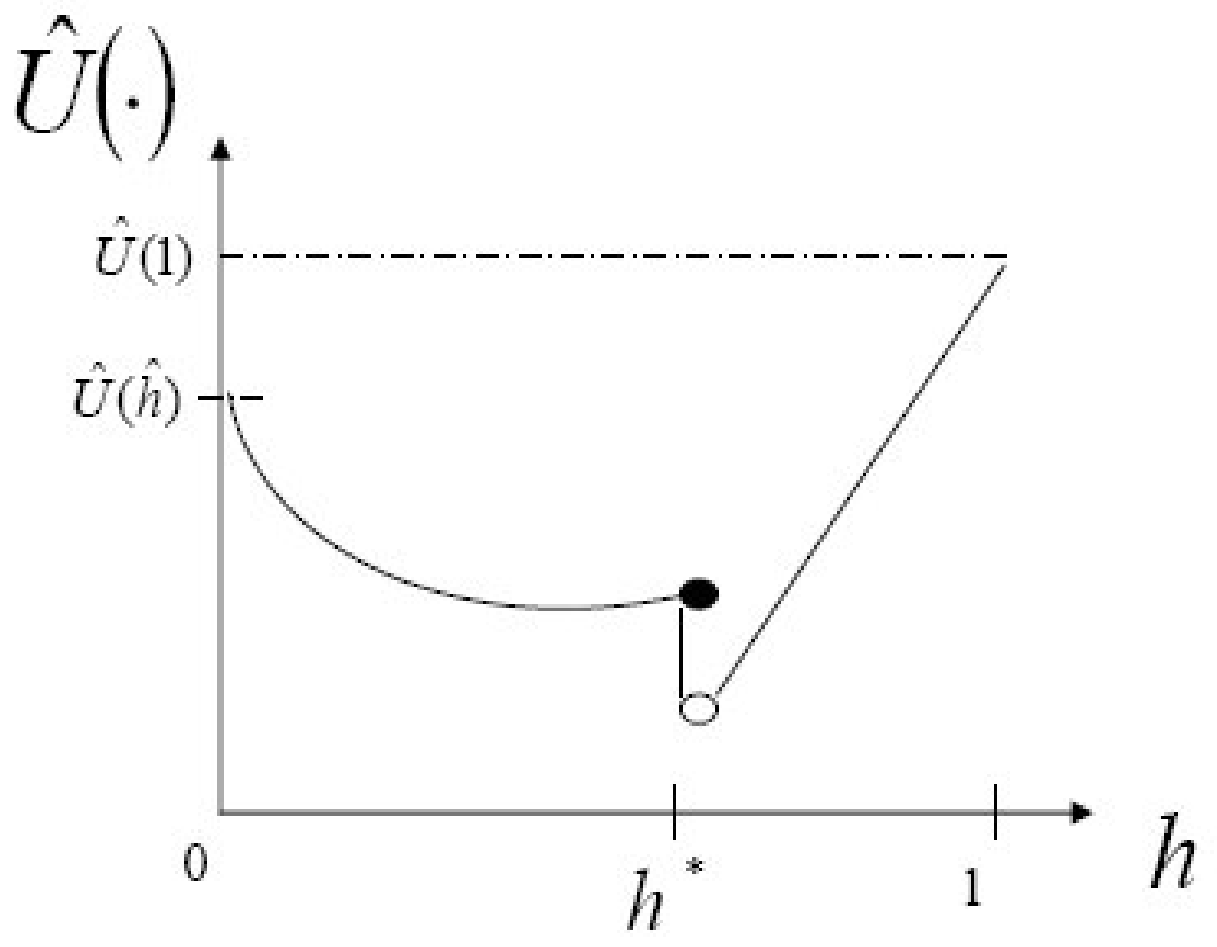

High Ability

Figure 5 


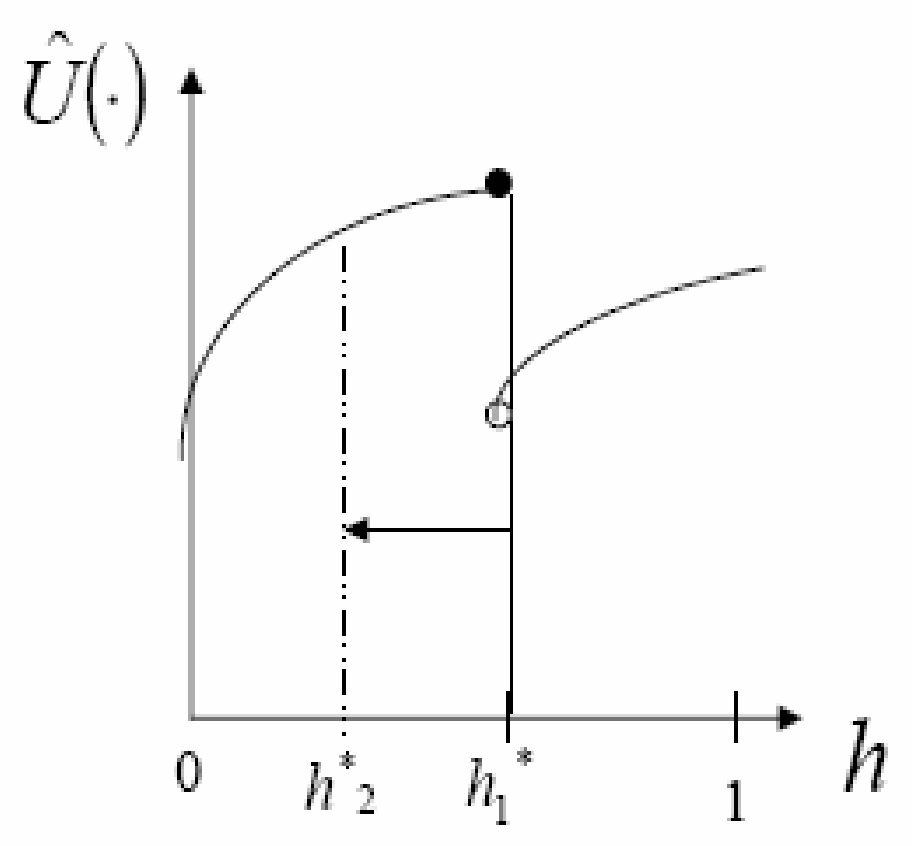

Low Ability

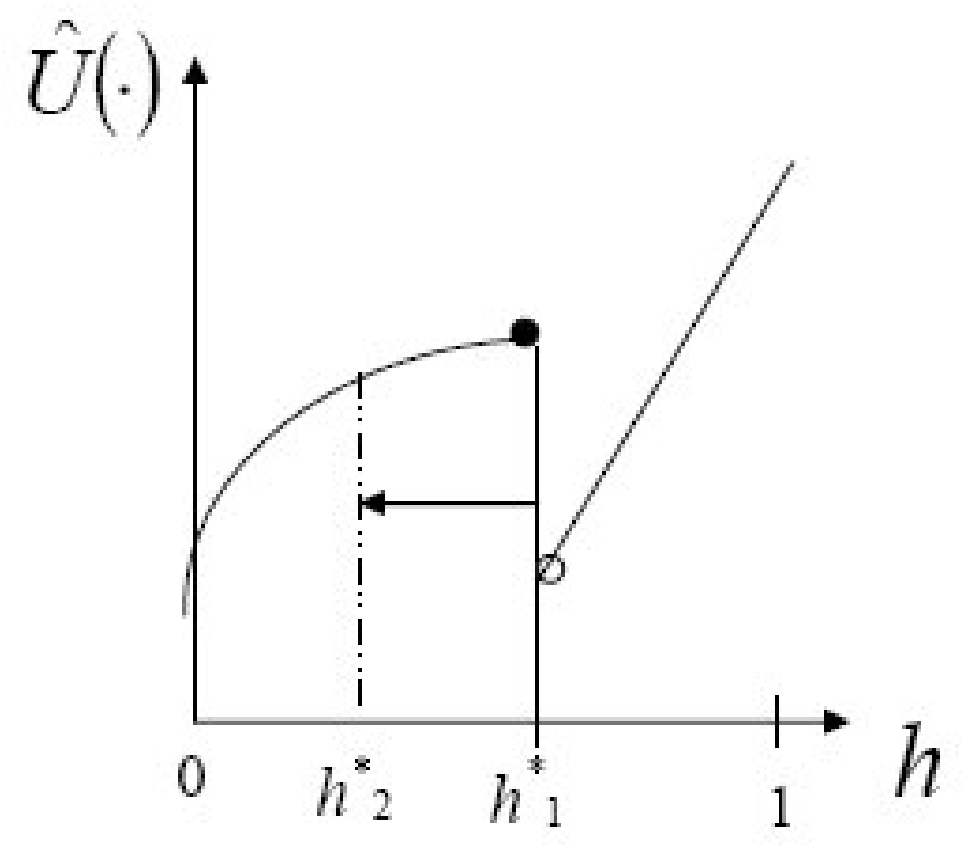

High Ability

Figure 6 


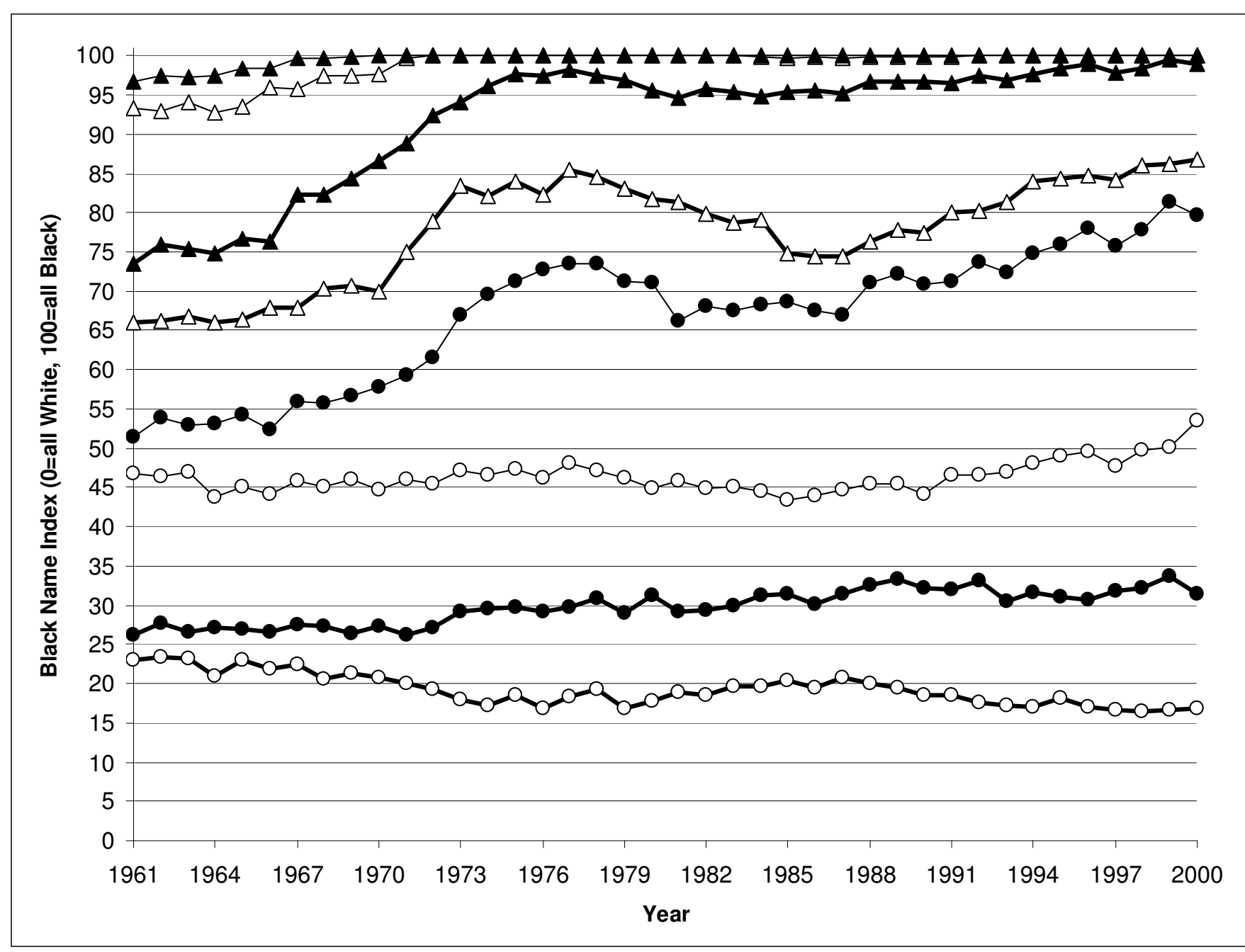

\}op Quartile

Second Quartile

Third Quartile

Bottom Quartile

O Blacks in Whitest Neighborhoods (10\% of babies born in Whitest

Hospitals)

- Blacks in Blackest Neighborhood (10\% of babies born in Blackest Hospitals)

Figure 7: Changes in Black Naming Patterns by Racial Composition of Neighborhood and by Quartile of Black Name Index 